\title{
Il decision making degli infermieri esperti e novizi nella struttura di dialisi peritoneale dell'Azienda Ospedaliera - IRCCS di Reggio Emilia. Uno Studio quali-quantitativo
}

\author{
Giovanna Amaducci ${ }^{1}$, Elena Baccarini ${ }^{2}$, Marina lemmi ${ }^{3}$, Emanuela Vezzani $^{4}$, Danio Somenzi ${ }^{5}$, Sonia Pasquali ${ }^{6}$ \\ ${ }^{1}$ Tutor e Docente di Infermieristica Generale al Corso di Laurea in Infermieristica, Sede di Reggio Emilia, Università degli studi di Modena e \\ Reggio Emilia, Reggio Emilia \\ ${ }^{2}$ Laureanda Corso di Laurea in Infermieristica, Sede di Reggio Emilia, Università degli studi di Modena e Reggio Emilia, Reggio Emilia \\ ${ }^{3}$ Direttore delle Professioni Sanitarie, Azienda Ospedaliera - IRCCS di Reggio Emilia, Reggio Emilia \\ ${ }^{4}$ Dirigente Infermieristico del Dipartimento di Medicina Interna e Specialità Mediche, Azienda Ospedaliera - IRCCS di Reggio Emilia, Reggio \\ Emilia \\ ${ }^{5}$ Dirigente di Struttura Complessa di Nefrologia e Dialisi, Azienda Ospedaliera - IRCCS di Reggio Emilia, Reggio Emilia \\ ${ }^{6}$ Direttore di Dipartimento di Medicina Interna e Specialità Mediche, Direttore di Struttura Complessa di Nefrologia e Dialisi, Azienda Os- \\ pedaliera - IRCCS di Reggio Emilia, Reggio Emilia
}

\begin{abstract}
Nurses decision making process in peritoneal dialysis
Purpose: Chronic kidney disease is now considered a major public health problem.

Over the past two decades, the number of patients that started dialysis is more than doubled.

Patient Management in Peritoneal Dialysis (PD) is considered as an advanced skill that requires technical technical competences, prevention and management of complications, as well as communicative-relational and educational knowledge to address patients and the caregiver needs.

In clinical settings, patient management in PD is entrusted to the experienced professional who becomes the central resource for the patient and for the interior care team.

In one type of such specialist care, it is important to understand what are the thought processes that guide the nurse expert decisions, so that the novice nurses through this explanation can learn about similar thought processes and enhance their expertise.

The study describes the different processes of thought, between expert and novice nurses, that guide the decision making in the management of patients in PD.

Methods: Quali-quantitative study, with simulated clinical cases.

Results: Experts gather complex data, focusing the relevant ones and possess perceptual acuity and effective observation skills. They make assumptions about possible problems, anticipate the presence and act with proactive mode. The novices collect a lot of data, less relevant, and monitor many parameters that do not know how to interpret. They make reference to general notions that do not correlate with the problem, find it difficult to set priorities and act in a reactive mode.

Conclusions: The study confirms the results of previous studies, however made in different clinical settings. The results obtained can be used for training courses for novice nurses for patient's management in PD, using the expertise of the expertise of competent professionals.
\end{abstract}

Keywords: Decision Making, Expert, Novice, Peritoneal dialysis

Accepted: February 6, 2017

Published online: August 31, 2017

Indirizzo per la corrispondenza:

Dr.ssa Emanuela Vezzani

Dipartimento di Medicina Interna e Specialità Mediche

Struttura Complessa di Nefrologia e Dialisi

Azienda Ospedaliera - IRCCS di Reggio Emilia

Viale Risorgimento, 80

42123 Reggio Emilia

emanuela.vezzani@asmn.re.it 


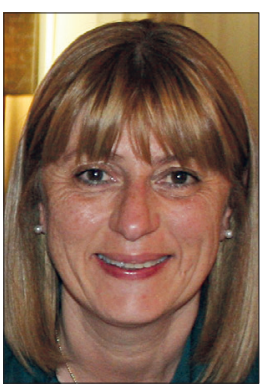

Emanuela Vezzani

\section{Introduzione}

La malattia renale cronica è oggi considerata un rilevante problema di salute pubblica. Si stima che nel mondo 1.800.000 pazienti siano in trattamento dialitico per uremia cronica e tale numero è in progressivo aumento. Anche in Italia, come nel resto del mondo, negli ultimi due decenni, il numero dei pazienti avviati alla dialisi è più che raddoppiato e continua ad aumentare (1).

L'aumento dell'età media dei pazienti uremici e la pluripatologia, insieme all'ampliamento dei setting assistenziali ai quali tali pazienti afferiscono, associati all'evoluzione della tecnologia e dei possibili trattamenti, ne hanno notevolmente accresciuto la complessità assistenziale (2).

Le unità operative di nefrologia e dialisi rappresentano un esempio di settore specialistico del lavoro infermieristico (3), nell'ambito del quale il professionista è chiamato a rispondere a complessi bisogni di salute (4).

La Dialisi Peritoneale (DP) rappresenta una delle metodiche di terapia sostitutiva per i pazienti con insufficienza renale in stadio avanzato. I pazienti con insufficienza renale cronica in fase sostitutiva avviati alla DP sono in costante aumento rispetto all'emodialisi extracorporea (5).

La gestione del paziente in DP è considerata una competenza avanzata che richiede abilità di tipo tecnico, di prevenzione e gestione delle complicanze, oltre che di tipo comunicativo-relazionale ed educativo, indirizzate al paziente e al caregiver $(6,7)$.

Nei contesti clinici, la gestione del paziente in DP è affidata al professionista esperto (Tab. I), che diviene, perciò, risorsa centrale per il paziente e per i colleghi neo-inseriti o di altri contesti assistenziali, i quali, non essendo in possesso delle competenze specifiche richieste, trovano difficoltà nella gestione di tali pazienti (8-10).

In un tipo di assistenza così specialistica, è importante comprendere quali sono i processi di pensiero che orientano le decisioni dell'infermiere esperto, affinché l'infermiere novizio, attraverso tale esplicitazione, possa apprendere processi di pensiero simili e accrescere la sua maturità professionale La maggior parte degli infermieri esperti ha poche opportunità per confrontarsi con altri infermieri e sviluppare il consenso attorno alla propria expertise, attraverso l'esplicitazione dei processi di pensiero che orientano il proprio agire (9-11).

Il decision making, come riportano Bakalis \& Watson (11), è fondamentale per la pratica infermieristica. L'infermiere è infatti il professionista più a stretto contatto con il paziente ed è perciò colui che più facilmente ne coglie i più piccoli cambiamenti nelle condizioni cliniche. L'interpretazione di tali modificazioni da parte dell'infermiere determina la successiva sequenza di azioni.

Mentre in letteratura sono numerosi gli studi in diversi ambiti clinici che fanno riferimento ai processi di pensiero che orientano la decisione clinica dell'infermiere esperto, pochi sono quelli relativi all'ambito di Nefrologia e Dialisi e, di questi, nessuno studio italiano.

\section{Decision making: modello teorico}

Tutti i modelli di pratica professionale si fondano essenzialmente su modelli decisionali ed essi stessi sono divenuti sinonimo di professionalità (12).

Il decision making è un processo che gli infermieri utilizzano quotidianamente quando esprimono giudizi circa l'assistenza da fornire ai pazienti e su come erogarla (12-14).

Cioffi (15) definisce il processo decisionale come un processo di pensiero discriminativo che viene utilizzato per scegliere una particolare linea di condotta. Bulfone (16) descrive il processo decisionale come il processo cognitivo che gli infermieri usano per raccogliere informazioni sui pazienti, generare ipotesi interpretative sul problema in atto, interpretare i dati raccolti alla luce delle ipotesi formulate e raccogliere ulteriori dati per validarle, identificare il problema assistenziale (diagnosi infermieristica) e mettere in atto una serie di interventi per la risoluzione del problema.

Si tratta di un processo cognitivo che coniuga conoscenze teoriche ed esperienza pratica (12).

In letteratura sono riportati diversi modelli interpretativi di tale processo cognitivo (12); il presente studio, sceglie di fare riferimento all'information-processing model e all'intuitivehumanist model, che rappresentano modelli fra loro integrati nel processo che porta alla presa di decisione $(12,16)$.

L'information-processing model (modello analitico) utilizza

TABELLA I - Livelli di competenza $(19,25)$

\begin{tabular}{ll}
\hline Esperto & Novizio \\
\hline - Infermiere con più di 3 anni di anzianità & - Infermiere con meno di 3 anni di anzianità \\
- Infermiere con più di 6 mesi di lavoro nell'unità di lavoro & - Infermiere di recente ingresso nell'unità operativa \\
- Infermiere riconosciuto dal gruppo come esperto & - Infermiere riconosciuto dal gruppo come novizio \\
- Infermiere che si considera esperto nell'assistenza alla tipologia & - Infermiere che si considera novizio nell'assistenza alla tipologia \\
di paziente. & di paziente. \\
\hline
\end{tabular}


l'approccio ipotetico-deduttivo alla presa di decisione e consta di quattro fasi: riconoscimento o acquisizione di informazioni, formulazione delle ipotesi, interpretazione delle informazioni e valutazione delle ipotesi $(12,13)$. Tale modello presuppone un continuo processo di raccolta e analisi dei dati che va dal particolare, il caso in esame, al generale, rappresentato dalle conoscenze possedute e dall'esperienza acquisita. La finalità di tale processo è rappresentata dalla validazione o dalla confutazione dell'ipotesi formulata (16).

In ambito infermieristico, la fase di acquisizione delle informazioni avviene durante l'incontro iniziale con il paziente, nel corso del quale l'infermiere raccoglie i dati. Segue la fase di formulazione delle ipotesi, alla luce delle quali interpreta $i$ dati raccolti. Tale interpretazione è finalizzata a confermare le ipotesi formulate e a scartare quelle non pertinenti. La fase finale consiste nell'individuazione di vantaggi e svantaggi di ogni alternativa possibile e nella scelta della migliore tra queste alternative per la risoluzione del problema. Nell'applicazione del processo ipotetico-deduttivo all'infermieristica viene identificata una fase aggiuntiva, relativa all'identificazione del problema assistenziale (diagnosi infermieristica) (12).

Nell'interpretazione del processo decisionale secondo il modello analitico, gli Autori riportano che questo è prevalentemente utilizzato nella sequenza sistematica delle sue fasi da infermieri novizi (17).

Gli infermieri esperti, secondo Hedberg e Larsson (18), invece, corroborano le loro ipotesi facendo riferimento al parere di colleghi, in particolare di quelli che possiedono una competenza specifica, per validare le proprie conoscenze e per l'interpretazione dei problemi assistenziali. II risultato di tale confronto fornisce spunti significativi per la presa di decisione in merito allo specifico problema. Il bisogno di confronto con il collega è spesso associato all'incertezza circa la decisione da prendere ed è funzionale per pervenire alla migliore decisione assistenziale.

Lo studio di Hedberg e Larsson (18) riporta inoltre che gli infermieri esperti, a partire dalla raccolta delle informazioni (anche solo di poche), sono in grado di anticipare i possibili problemi e di adottare strategie per prevenire i problemi anticipati; essi agiscono, cioè, in maniera proattiva. Tale abilità è da correlarsi al fatto che gli infermieri, a partire dalla loro esperienza, sono in grado di riconoscere situazioni assistenziali simili e possibili eventi avversi e sono perciò incoraggiati ad agire in modo autonomo. I principianti, invece, mettono in atto il processo decisionale, quando i problemi sono già insorti, agiscono cioè con modalità reattiva; questo poiché non hanno esperienze che gli consentano di prefigurarsi il corso degli eventi, dunque agiscono prevalentemente su prescrizione.

L'intuitive-humanistic model (modello intuitivo) ha come focus l'intuizione e la relazione di questa con l'esperienza, la conoscenza acquisita attraverso di essa e la crescita del processo decisionale via via che l'infermiere accresce la sua esperienza professionale. In questo modello, la validazione delle ipotesi non avviene attraverso un ragionamento scientifico ma si fonda sull'intuizione, cosa che ha reso molti Au- tori scettici circa la validità di tale processo decisionale. Tale scetticismo però non è motivato, se si pensa al fatto che l'infermiere deve fare fronte costantemente a situazioni cliniche che si caratterizzano per un'elevata instabilità e soggette a rapide modificazioni, per far fronte alle quali fondamenti razionali e scientifici risultano, talvolta, inadeguati e insufficienti (12).

Secondo Benner, mentre l'infermiere novizio, nella presa di decisioni, si avvale di un processo razionale fondato sui principi forniti da Linee Guida e procedure, con l'acquisizione di esperienza, il processo decisionale si fonda sull'intuizione (19).

Numerosi Autori hanno cercato di definire l'intuizione (12): secondo Benner e Tanner è una comprensione senza un fondamento logico, la conoscenza di un fatto o di una verità nel suo complesso e l'immediata detenzione di conoscenza, che è indipendente dal processo di ragionamento lineare, logico e analitico (20).

L'intuizione è stata definita da Rew (21) anche come la conoscenza immediata di qualcosa senza l'uso consapevole della ragione. Inoltre, secondo il medesimo Autore, I'intuizione è una componente del giudizio complesso e l'atto di decidere cosa fare di fronte a una situazione ambigua, incerta e improvvisa. È frutto di una sintesi di conoscenze empiriche, etiche, estetiche e personali. È la decisione di agire a partire da un'improvvisa consapevolezza cognitiva, difficile da esplicitare, che deriva da precedenti esperienze con situazioni assistenziali simili.

Alcuni Autori infatti correlano l'intuizione con l'esperienza. Secondo King \& Clark (22) la qualità di analisi del processo decisionale migliora con l'esperienza, inoltre Watson sostiene che l'esperienza aumenta la facilità con la quale l'infermiere prende decisioni (23).

Secondo Hedberg and Larsson (18) il decision making dell'infermiere esperto si fonda sull'identificazione di indizi, identificazione che è supportata dalla conoscenza del paziente e dalle conoscenze proprie dell'infermiere. Tali conoscenze aiutano l'infermiere esperto a riconoscere e a confrontare gli indizi raccolti con quelli precedentemente incontrati sullo stesso paziente o su pazienti simili.

L'abilità dell'infermiere nel processo di riconoscimento di indizi significativi si accresce con l'aumentare di conoscenze ed esperienza su determinate tipologie di pazienti e di ambito assistenziale. Con il passare del tempo tale abilità di riconoscimento diventa sempre più raffinata e affidabile, cosa che è associata a competenza, a diminuzione dell'ansia e a un senso di padronanza della situazione che è una caratteristica propria dell'infermiere esperto $(12,20,24)$.

L'infermiere esperto percepisce la situazione come un insieme, utilizza le situazioni vissute in passato come paradigmi e analizza il problema senza perdere tempo a considerare indizi non significativi. II processo decisionale dell'infermiere esperto si fonda su tale comprensione intuitiva della situazione che gli consente di focalizzare rapidamente il problema a partire da pochi indizi senza perdere tempo a raccogliere dati non necessari. Pertanto la concreta esperienza fatta in passato guida le percezioni e il processo decisionale dell'esperto, 
TABELLA II - Caso simulato suddiviso nei 2 Step (16)

Il caso clinico simulato suddiviso negli Step

Step 1

Il Signor FD di 78 anni entra in Nefrologia per ipertensione arteriosa mal controllata, refrattaria alla terapia farmacologica. Dall'anamnesi risulta che è affetto da insufficienza renale cronica di grado severo per una nefropatia diabetica, in trattamento dialitico peritoneale da 5 anni, con due ricoveri nell'ultimo anno per peritonite. All'ingresso presenta PA in clinostatismo 150/90 mmHg, PA in ortostatismo $135 / 100$ $\mathrm{mmHg}$, respiro eupnoico e peso $90.1 \mathrm{Kg}$. Obiettivamente scarse masse muscolari e ridotti depositi di grasso. Riferisce una diuresi ridotta nelle ultime settimane, con comparsa di edemi declivi di grado moderato, in aumento nonostante la terapia diuretica recentemente aumentata su indicazione del Nefrologo. Riferisce lieve addominalgia dal mattino.

- Quali dati raccoglie nella situazione presentata?

- Quali problemi assistenziali ipotizza?

- Quali ipotesi formula sulla causa dei problemi identificati?

- Che cosa decide di fare (in autonomia e/o in collaborazione) per dare risposta ai problemi identificati?

Step 2

Sono le h. 05.00 e FD sta facendo la seduta di dialisi peritoneale dalla sera precedente. La chiama perché avverte dolore. Lo descrive come pungente, localizzato in addome, ma irradiato a tutti i quadranti.

- Quali dati raccoglie nella situazione presentata?

- Che cosa pensa stia succedendo (ipotesi sui possibili problemi)?

- Che cosa decide di fare (in autonomia e/o in collaborazione) per dare risposta ai problemi ipotizzati?

permettendogli una rapida comprensione percettiva della situazione (20-22, 24, 25).

Scopo del presente studio è di descrivere le differenti modalità nella presa di decisione, fra infermiere novizio ed esperto, nella gestione del paziente in dialisi peritoneale, attraverso l'esplicitazione dei processi di pensiero che orientano l'agire.

\section{Metodi}

Studio descrittivo, che utilizza un metodo quali-quantitativo. Lo strumento utilizzato per analizzare la capacità di decision making degli infermieri novizi ed esperti è un caso simulato (Tab. II) di una situazione problematica relativa a un paziente in dialisi peritoneale suddiviso in 2 step. La finalità di ciascuno dei 2 step è quella di identificare i problemi assistenziali secondo priorità, di formulare ipotesi sulle cause dei problemi, di raccogliere dati e di descrivere gli interventi attuati per la risoluzione dei problemi (16).

Affinché il caso sia il più realistico possibile, ci si è avvalsi, per la sua elaborazione, della collaborazione di un medico della Struttura Complessa di Nefrologia e Dialisi dell'Azienda Ospedaliera - IRCCS di Reggio Emilia. Non è stata chiesta, invece, la collaborazione del coordinatore infermieristico, per evitare di influenzare gli infermieri coinvolti nello studio e gli infermieri poiché parti in causa dello studio in oggetto.

Il setting dello studio è la Struttura Complessa di Nefrologia dell'Azienda Ospedaliera - IRCCS di Reggio Emilia nel periodo Giugno-Luglio 2015.

I partecipanti allo studio sono i 9 infermieri della Struttura. Tutti sono stati preliminarmente contattati, è stata spiegata loro la finalità dello studio ed è stato chiesto il consenso alla partecipazione.

Il protocollo dello studio è stato inviato per parere al Comitato Etico Provinciale per "garantire la tutela dei diritti, della sicurezza e del benessere dei soggetti della sperimentazione e per fornire pubblica garanzia di questa tutela" (Direttiva 2001/20/CE del Parlamento Europeo) e al Comitato Tecnico Scientifico dell'Azienda Ospedaliera - IRCCS.

I dati sono stati raccolti attraverso la somministrazione ai professionisti di un caso simulato in 2 step successivi della durata di circa 15 minuti ciascuno.

Per ogni step, ciascun professionista singolarmente riporta in modo scritto le valutazioni (ipotesi esplicative, dati significativi, interventi da porre in essere) sulla situazione assistenziale proposta.

Prima di passare allo step successivo, è stato chiesto a ciascun professionista di presentare verbalmente (pensare a voce alta) quanto scritto, per dar loro la possibilità di esplicitare il processo mentale seguito, e ai ricercatori di prendere nota di tale processo.

L'incontro è stato inoltre audio-registrato previo consenso da parte dei partecipanti, attraverso l'utilizzo di un registratore vocale, allo scopo di ridurre gli errori di analisi e di aumentare l'affidabilità nel processo di elaborazione dei dati.

Al termine dell'incontro sono stati ritirati gli "elaborati" scritti quali fonti di ulteriori dati, chiedendo agli infermieri di identificarsi come esperti o novizi.

I dati, raccolti attraverso sbobinatura delle registrazioni e lettura degli scritti, sono stati trascritti e analizzati secondo la tecnica di analisi del contenuto (25). Sono stati successivamente inseriti in un database e aggregati nei tre gruppi, rispettivamente, esperti e novizi. 
TABELLA III - STEP 1 Sintesi dati raccolti, problemi identificati, cause ed interventi attuati da esperti e novizi

\begin{tabular}{|c|c|c|c|c|c|c|c|c|c|c|c|c|c|c|c|c|}
\hline & $\begin{array}{c}\text { A } \\
\text { Dati raccolti }\end{array}$ & 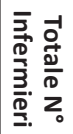 & 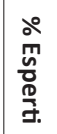 & $\begin{array}{l}\text { ஃo } \\
2 \\
\text { o } \\
\text { N. }\end{array}$ & $\begin{array}{c}\text { B } \\
\text { Problemi }\end{array}$ & 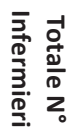 & 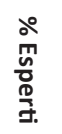 & 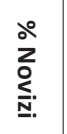 & $\begin{array}{c}\text { C } \\
\text { Cause }\end{array}$ & 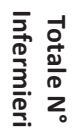 & 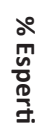 & $\begin{array}{l}\text { o̊ } \\
\text { Z } \\
\text { L. } \\
\text { N. }\end{array}$ & $\begin{array}{c}\text { D } \\
\text { Interventi/ } \\
\text { Decisioni }\end{array}$ & 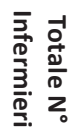 & 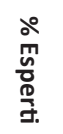 & 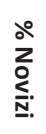 \\
\hline \multirow{6}{*}{ 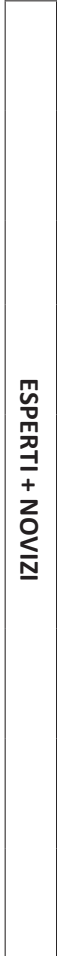 } & $\begin{array}{l}\text { Pressione } \\
\text { arteriosa }\end{array}$ & 6 & 50 & 100 & Addominalgia & 9 & 100 & 100 & Peritonite & 6 & 67 & 67 & $\begin{array}{l}\text { Chiamare/ } \\
\text { collaborare } \\
\text { con il medico }\end{array}$ & 7 & 100 & 33 \\
\hline & Peso & 5 & 67 & 33 & Edemi declivi & 3 & 33 & 33 & $\begin{array}{l}\text { Dieta non } \\
\text { adeguata }\end{array}$ & 2 & 17 & 33 & $\begin{array}{l}\text { Eseguire RX } \\
\text { torace/ addome }\end{array}$ & 4 & 33 & 67 \\
\hline & Diuresi & 5 & 33 & 100 & $\begin{array}{l}\text { Aumento } \\
\text { ponderale }\end{array}$ & 3 & 33 & 33 & $\begin{array}{l}\text { Insuff Renale } \\
\text { Cronica }\end{array}$ & 2 & 17 & 33 & $\begin{array}{l}\text { Modificare dieta in } \\
\text { iposodica/ } \\
\text { iperproteica }\end{array}$ & 4 & 50 & 33 \\
\hline & $\begin{array}{l}\text { Parametri } \\
\text { vitali }\end{array}$ & 3 & 33 & 33 & & & & & Edemi declivi & 2 & 17 & 33 & $\begin{array}{l}\text { Somministrare } \\
\text { terapia } \\
\text { antibiotica }\end{array}$ & 3 & 33 & 33 \\
\hline & $\begin{array}{l}\text { Temperatura } \\
\text { corporea }\end{array}$ & 3 & 33 & 33 & & & & & $\begin{array}{l}\text { Diuresi } \\
\text { ridotta/ } \\
\text { assente }\end{array}$ & 2 & 17 & 33 & $\begin{array}{l}\text { Consigliare la } \\
\text { restrizione idrica }\end{array}$ & 3 & 33 & 33 \\
\hline & $\begin{array}{l}\text { Valutazione } \\
\text { caratteristiche } \\
\text { urine }\end{array}$ & 2 & 17 & 33 & & & & & $\begin{array}{l}\text { Ritenzione di } \\
\text { liquidi }\end{array}$ & 2 & 17 & 33 & & & & \\
\hline
\end{tabular}

\section{Risultati}

\section{Caratteristiche del campione}

Per evitare una dispersione di dati, il campione degli infermieri è stato suddiviso in infermieri esperti (6/9) e in infermieri novizi (3/9), secondo i criteri riportati nella Tabella I.

Il campione dello studio è composto da 2 maschi e 7 femmine. I partecipanti hanno un range di età compreso fra i 29 e i 53 anni, i 6 esperti hanno un'età media pari a 45 anni e i novizi pari a 43 anni. Gli esperti hanno un'anzianità di servizio in nefrologia compresa fra i 5 e i 37 anni, mentre i novizi fra i 2 e i 4 mesi. Degli esperti nessuno ha un titolo di formazione post base, mentre 1 novizio ha il titolo di Laurea magistrale (Tab. III).

\section{STEP 1 (Tab. I)}

\section{Dati raccolti}

Gli esperti monitorano in media 5.7 (min. 4-max. 9) parametri, mentre i novizi 4.7 (min. 3-max. 6).

Nelle Tabelle III, IV e V colonna A il dettaglio dei dati raccolti rispettivamente da esperti e novizi.

\section{Problemi identificati}

Le problematiche individuate mediamente sono state: 3.5 (min. 3-max. 5) per gli esperti e 5 (min. 4-max. 7) per i novizi.

Nelle Tabelle III, IV e V colonna B il dettaglio dei problemi identificati rispettivamente da esperti e novizi.

\section{Ipotesi sulle cause dei problemi}

Le ipotesi sulle cause dei problemi che gli infermieri hanno individuato sono: in media 4.5 (min. 1-max. 7) per gli esperti e 4 (min. 0-max. 8) per i novizi.

Nelle Tabelle III, IV e V colonna C il dettaglio dei dati raccolti rispettivamente da esperti e novizi.

\section{Interventi attuati/decisioni assunte}

Gli interventi attuati in risposta ai problemi identificati e ai dati raccolti sono in media 9 per gli esperti (min. 6-max. 12) e 4.7 per i novizi ( $\min .2$-max. 7).

Nelle Tabelle III, IV e V colonna D il dettaglio degli interventi attuati/decisioni assunte rispettivamente da esperti e novizi. 
TABELLA IV - STEP 1 Sintesi dati raccolti, problemi identificati, cause ed interventi attuati da esperti

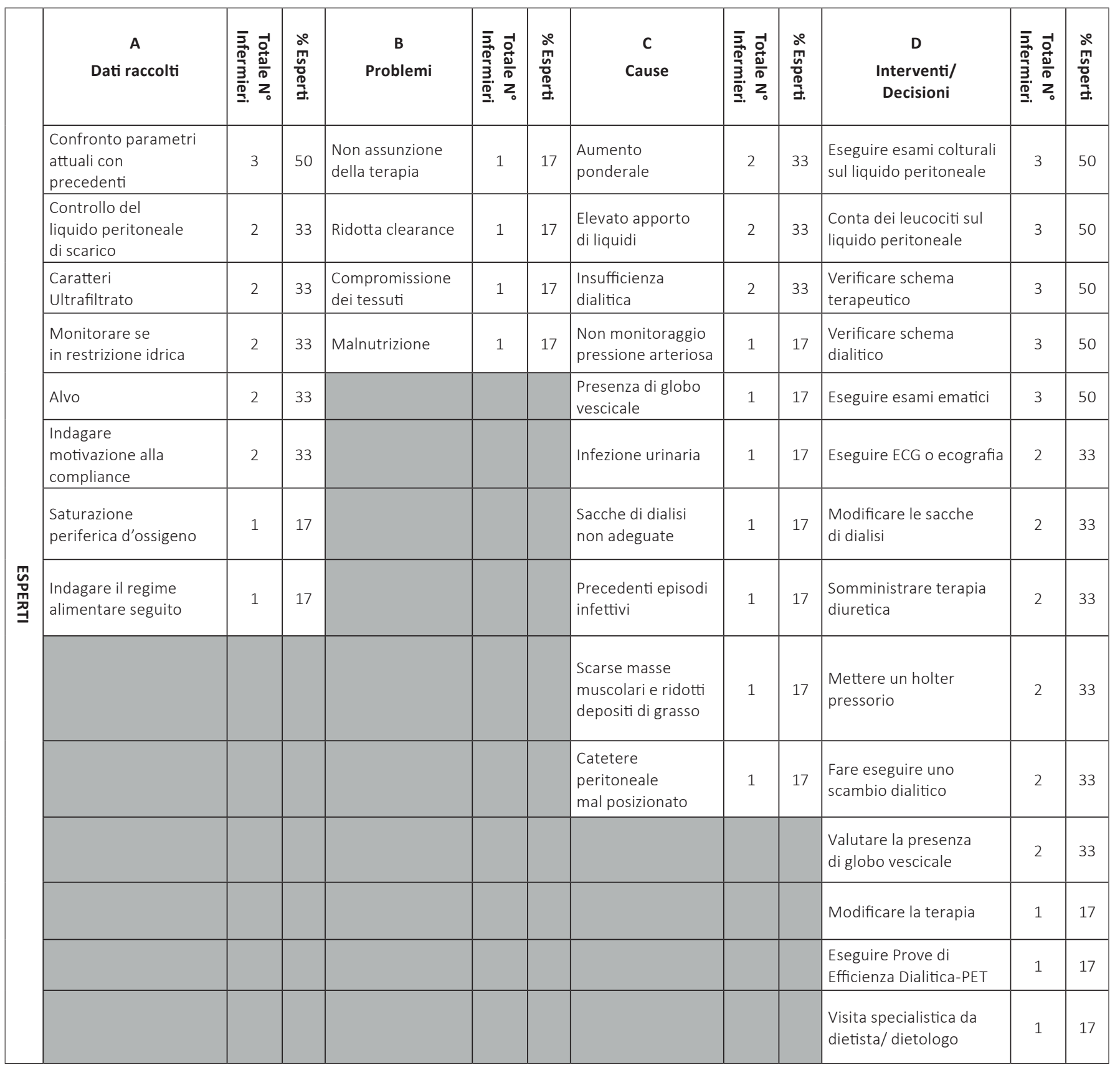

STEP 2 (Tab. II)

\section{Dati raccolti}

Gli infermieri esperti rilevano in media 4.8 (min. 3-max. 12) dati, mentre i novizi 1.3 (min. 0-max. 4) (Tab. VI).

In Tabella VI colonna A il dettaglio dei dati raccolti da esperti e novizi.

\section{Ipotesi sui possibili problemi}

Gli infermieri esperti ipotizzano mediamente 3.2 (min. 1-max. 5) problemi possibili, mentre i novizi 1 ( $\min .0$-max. 3).

In Tabella VI colonna B e C il dettaglio delle ipotesi sui possibili problemi formulate da esperti e novizi. 
TABELLA V - STEP 1 Sintesi dati raccolti, problemi identificati, cause ed interventi attuati dai novizi

\begin{tabular}{|c|c|c|c|c|c|c|c|c|c|c|c|c|}
\hline & $\begin{array}{c}\text { A } \\
\text { Dati raccolti }\end{array}$ & 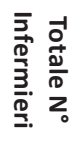 & $\begin{array}{l}\stackrel{0}{0} \\
2 \\
o \\
\grave{N} .\end{array}$ & $\begin{array}{c}\text { B } \\
\text { Problemi }\end{array}$ & 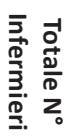 & $\begin{array}{l}\text { do } \\
2 \\
0 \\
\text { … }\end{array}$ & $\begin{array}{c}\text { C } \\
\text { Cause }\end{array}$ & 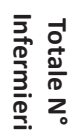 & $\begin{array}{l}\text { do } \\
\text { Z } \\
\text { o } \\
\text { N. }\end{array}$ & $\begin{array}{c}\text { D } \\
\text { Interventi/ } \\
\text { Decisioni }\end{array}$ & 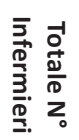 & $\begin{array}{l}\text { do } \\
2 \\
0 \\
\text { L. } \\
\text { N. }\end{array}$ \\
\hline \multirow{5}{*}{ 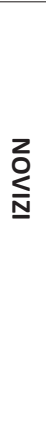 } & $\begin{array}{l}\text { Nessun dato } \\
\text { ulteriore }\end{array}$ & 3 & 100 & $\begin{array}{c}\text { Insufficienza Renale } \\
\text { Cronica }\end{array}$ & 2 & 67 & $\begin{array}{l}\text { Nefropatia } \\
\text { diabetica }\end{array}$ & 1 & 33 & $\begin{array}{c}\text { Posizionare il paziente } \\
\text { con arti in scarico }\end{array}$ & 2 & 67 \\
\hline & & & & Diabete & 2 & 67 & $\begin{array}{l}\text { Ipertensione } \\
\text { arteriosa }\end{array}$ & 1 & 33 & $\begin{array}{l}\text { Somministrare terapia } \\
\text { ipoglicemizzante }\end{array}$ & 1 & 33 \\
\hline & & & & Peritonite & 1 & 33 & $\begin{array}{l}\text { Non efficacia } \\
\text { della terapia }\end{array}$ & 1 & 33 & Consigliare il digiuno & 1 & 33 \\
\hline & & & & & & & $\begin{array}{c}\text { Non conoscere } \\
\text { le cause }\end{array}$ & 1 & 33 & $\begin{array}{c}\text { Cambiare decubito } \\
\text { al paziente }\end{array}$ & 1 & 33 \\
\hline & & & & & & & & & & Eseguire emocolture & 1 & 33 \\
\hline
\end{tabular}

\section{Interventi attuati/decisioni assunte}

Gli interventi attuati, sulla base dei possibili problemi, sono mediamente 4.7 ( $\min .3-\max .8$ ) per gli esperti e 0.7 (min. 0-max. 2) per i novizi.

In Tabella VI colonna D il dettaglio degli interventi attuati/ decisioni assunte da esperti e novizi.

\section{Discussione}

Lo studio descrive le differenti modalità nella presa di decisione, fra infermiere novizio ed esperto, nella gestione del paziente in dialisi peritoneale, attraverso l'esplicitazione dei processi di pensiero che orientano l'agire nella gestione di un caso simulato.

I parametri rilevati e i dati raccolti dagli infermieri esperti sono relativamente numericamente superiori, ma più specifici, rispetto alla tipologia di paziente e alla sintomatologia riferita, infatti, in accordo con quanto scritto da Benner (19) e da Bulfone et al. (16), gli esperti possiedono acutezza percettiva, capacità di riconoscimento ed efficaci abilità di osservazione.

I novizi, non avendo conoscenze specifiche, raccolgono dati generali e aspecifici. Per poter acquisire acutezza percettiva sono infatti necessarie conoscenze specifiche oltre che una ripetuta esperienza su tale tipologia di pazienti. La formazione infermieristica di base non fornisce conoscenze e abilità sufficienti per la gestione di pazienti in DP, in quanto argomenti specialistici non facenti parte del percorso formativo di base (10).

Hoffman, Aitken, Duffield (25) sostengono infatti che esperti e novizi raccolgano entrambi molti dati, ma mentre i primi identificano dati più complessi e riescono più facilmente a focalizzarsi sugli aspetti principali senza perdere di vista le informazioni rilevanti, i novizi, invece, raccolgono molti dati, meno rilevanti e non li graduano per importanza.

Gli esperti, inoltre focalizzano ulteriori dati rilevanti e ne specificano il loro significato rispetto ai possibili problemi, nei termini di "Controllo l'alvo [...] ha avuto diarrea? $\dot{E}$ un indice di peritonite", "... cercherei il valore del peso di un controllo precedente per valutare se può avere accumulato dei liquidi. Ma comunque il paziente è eupnoico, per cui, se ne ha accumulati, i livelli non sono tali da causargli difficoltà respiratorie...", "controllerei il liquido peritoneale e i tempi che impiega per il carico e lo scarico [...] mi serve per capire se funziona come meccanica", "[...] controllo la glicemia perché il paziente è diabetico e se fa il glucosio nelle sacche potrebbe aumentare $[. . .]^{\prime \prime}$.

Come riportato da Benner (19) e da Hoffman, Aitken, Duffield (25), gli esperti, a partire da indizi che per un professionista non esperto possono apparire non significanti, possono aver idea del problema e della sua gravità.

Inoltre, durante lo studio, un esperto dice "sarebbe interessante avere altri dati che però mancano", evidenziando, da un lato, il limite dell'utilizzo di un caso simulato, dall'altro il fatto che, di fronte a un paziente reale, l'abilità dell'esperto si sarebbe manifestata anche attraverso il monitoraggio di "altro" in relazione a ipotesi circa possibili evoluzioni nelle condizioni cliniche del paziente (modalità proattiva) (25).

Gli esperti identificano problemi, molto specifici rispetto al caso descritto, quali: "ridotta clearance", "possibile non assunzione della terapia", "eventuale malnutrizione".

I novizi, invece, a causa della loro poca esperienza con pazienti simili, tendono a fare riferimento a problemi molto generali e ascrivibili a diagnosi mediche, quali: "insufficienza renale cronica", "diabete", "peritonite", che risentono di un forte ancoraggio a conoscenze teoriche più che a esperienze vissute.

Le cause correlate ai problemi vengono ipotizzate da en- 


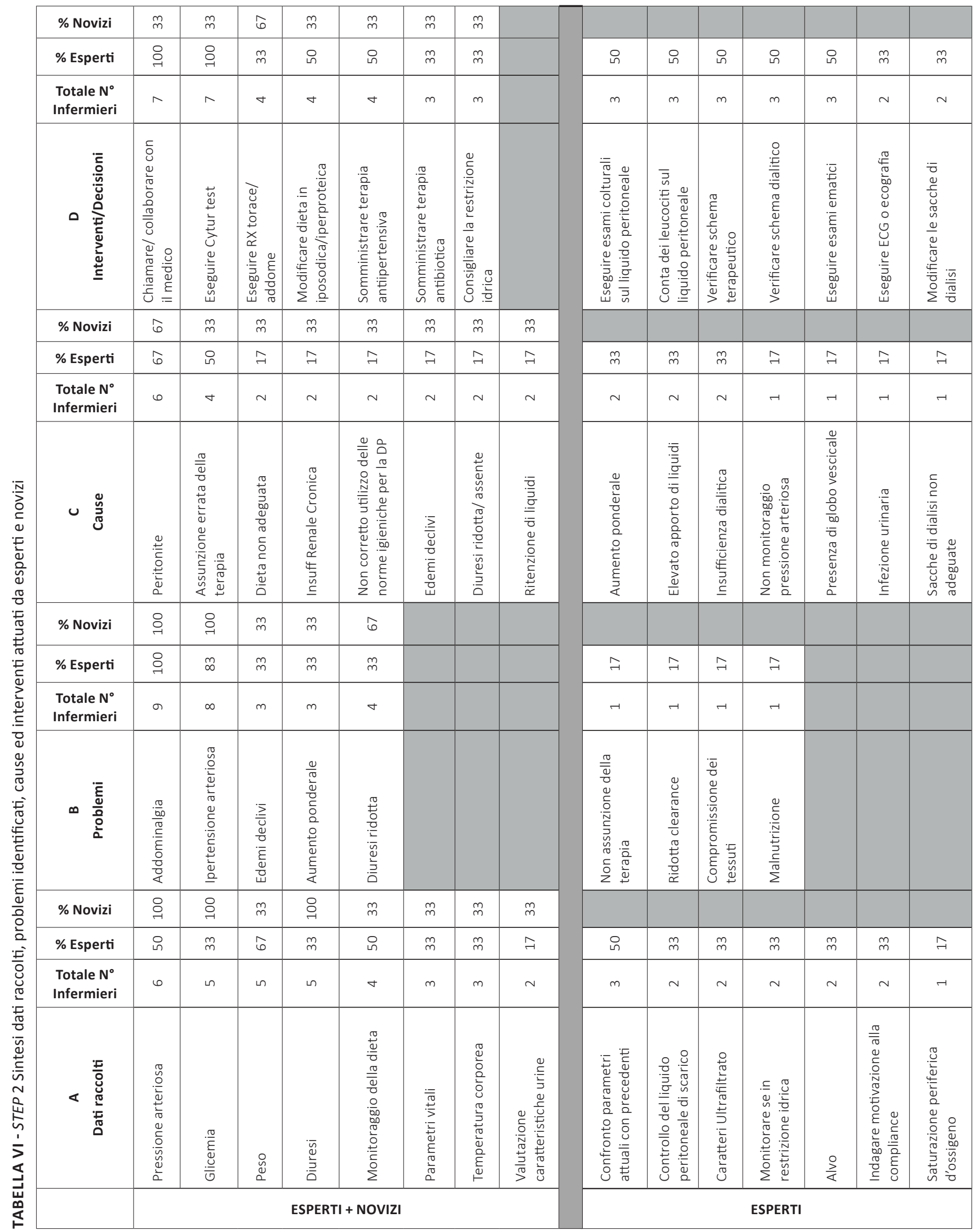




\begin{tabular}{|c|c|c|c|c|c|c|c|c|c|c|c|c|}
\hline \% Novizi & & & & & & & & $\widehat{6}$ & $m$ & $m$ & $m$ & $m$ \\
\hline \% Esperti & $m$ & $\stackrel{m}{m}$ & $\stackrel{m}{m}$ & $\stackrel{m}{m}$ & न & ને & ન & & & & & \\
\hline $\begin{array}{l}\text { Totale } \mathbf{N}^{\circ} \\
\text { Infermieri }\end{array}$ & $\sim$ & $\sim$ & $\sim$ & $\sim$ & - & -1 & - & $\sim$ & -1 & $r$ & -1 & -1 \\
\hline ○ & 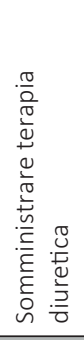 & 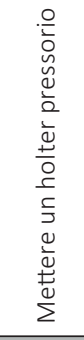 & 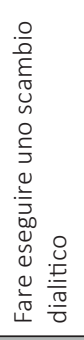 & 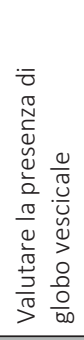 & 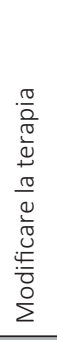 & 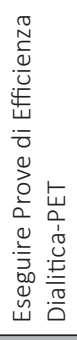 & 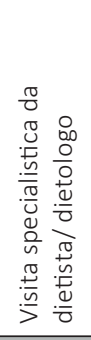 & 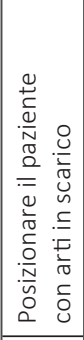 & 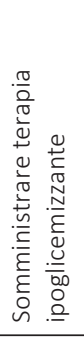 & 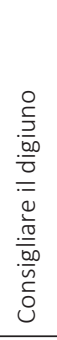 & 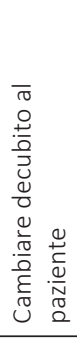 & 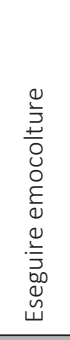 \\
\hline \% Novizi & & & & & & & & $\stackrel{m}{m}$ & $\stackrel{m}{m}$ & $\stackrel{m}{m}$ & $\stackrel{m}{m}$ & \\
\hline \% Esperti & न & न & 군 & & & & & & & & & \\
\hline $\begin{array}{l}\text { Totale } \mathbf{N}^{\circ} \\
\text { Infermieri }\end{array}$ & -1 & $\neg$ & $r$ & & & & & -1 & - & $r$ & $\rightarrow$ & \\
\hline ৩ & 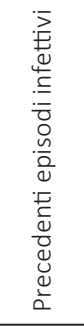 & 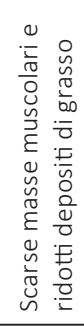 & 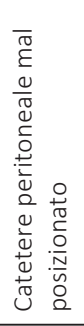 & & & & & 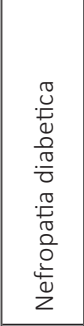 & 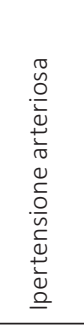 & 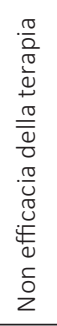 & 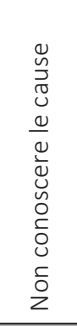 & \\
\hline \% Novizi & & & & & & & & $\hat{6}$ & $\hat{\sigma}$ & $\stackrel{m}{m}$ & & \\
\hline \% Esperti & & & & & & & & & & & & \\
\hline $\begin{array}{l}\text { Totale } \mathbf{N}^{\circ} \\
\text { Infermieri }\end{array}$ & & & & & & & & $\sim$ & $\sim$ & $-r$ & & \\
\hline$\infty \frac{\overline{\bar{\varepsilon}}}{\frac{\bar{c}}{0}}$ & & & & & & & & 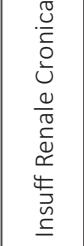 & 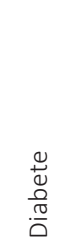 & 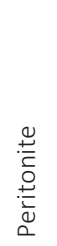 & & \\
\hline \% Novizi & & & & & & & & & & & & \\
\hline \% Esperti & 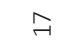 & & & & & & & & & & & \\
\hline $\begin{array}{l}\text { Totale } \mathbf{N}^{\circ} \\
\text { Infermieri }\end{array}$ & - & & & & & & & & & & & \\
\hline 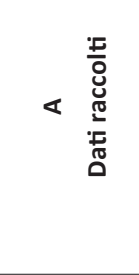 & 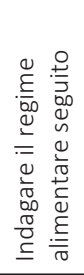 & & & & & & & & & & & \\
\hline & \multicolumn{7}{|c|}{ ESPERTI } & \multicolumn{5}{|c|}{ NOVIZI } \\
\hline
\end{tabular}


trambi i gruppi, in numero non significativamente maggiore dagli esperti rispetto ai novizi.

Gli esperti però sono in grado di ipotizzare cause molto specifiche sottese ai problemi identificati, quali: "insufficienza dialitica", "presenza di globo vescicale" "catetere peritoneale mal posizionato", "sacche di dialisi non adeguate", mentre i novizi invece identificano cause molto più generali: "nefropatia diabetica", "ipertensione arteriosa", "non efficacia della terapia" (19).

Gli esperti, possedendo un notevole background di esperienze con pazienti simili, oltre a raccogliere un numero maggiore di dati, sono anche più accurati nella selezione e nella correlazione con il problema.

Tutti gli infermieri (novizi ed esperti) mettono in atto interventi coerenti con i problemi identificati, mentre uno solo dei novizi riporta, anche, che, degli interventi attuati, però, non ne conosce a fondo il razionale. Quest'ultimo aspetto è coerente con quanto riportato in letteratura, infatti, Hancock \& Easen (2006) (26) nel loro studio riportano che i novizi, all'inizio dell'attività nel nuovo setting di lavoro, spesso non ritengano una priorità comprendere la motivazione degli interventi infermieristici messi in atto, ma che, piuttosto, preferiscano agire "come si usa fare" dagli infermieri esperti del contesto, questo anche per essere accettati meglio dal nuovo gruppo di lavoro.

Gli interventi attuati dagli esperti sono numericamente superiori. La diversa numerosità di interventi è caratteristica propria dell'expertise, ossia della consuetudine con una certa tipologia di pazienti. Inoltre l'esperto, sulla base dell'intuizione, mette in atto una serie di interventi, l'esito dei quali conferma, al massimo, la correttezza dell'intuizione $(20,21)$.

I novizi, non avendo esperienza con la tipologia di pazienti come quella del caso, tendono a essere molto vaghi e generici nella proposta di interventi: "non conosco gli interventi da attuare, se non iniziare una terapia antibiotica e consigliare il digiuno" e "cambiare decubito al paziente".

Gli esperti, inoltre, come riportano Bonner \& Walker (4), spesso, al fine di prevenire ulteriori complicanze, sono portati non solo ad attuare interventi appropriati e specifici per il proprio ambito di competenza, quali: "inizialmente faccio fare uno scarico del liquido peritoneale [...]", "verifico lo schema terapeutico e se è compliante per l'assunzione della terapia", ma anche ad anticipare o comunque a pensare a interventi, al di fuori delle proprie aree di competenza: "si fanno gli esami colturali sul liquido peritoneale [...], poi si manda in laboratorio", "cambiare la concentrazione delle sacche di dialisi per aumentare l'ultrafiltrato e quindi diminuire il peso della persona", "programmo gli esami per poi iniziare la terapia antibiotica", "faccio un'ecografia vescicale" e così via.

Relativamente alla gestione della complicanza presentata nello step 2, il $67 \%(2 / 3)$ dei novizi ha detto che "non metterebbe in atto alcun intervento". Questa affermazione è coerente con quanto espresso da Dìez de Baldeòn, Cidoncha \& Gistau (2003) (27): gli infermieri novizi non possiedono competenze cliniche specifiche e perciò non sanno, rispetto all'imprevisto, quali interventi mettere in atto. Al contrario, gli esperti sono molto specifici nella proposta di interventi: "faccio girare il paziente su entrambi i lati [...] se tutto ciò non dà beneficio faccio altre ipotesi", "valuto [...] per vedere se è quello il problema oppure no", "[...] quindi fermarsi è la prima cosa, perché, se si tratta di questo problema, gli fai anche passare il dolore [...]", "se il liquido mi dà idea di assenza di peritonite sospendo la dialisi [...]". L'intervento del medico viene richiesto perlopiù dopo un'attenta valutazione del paziente, per quanto riguarda l'esperto; il novizio, invece, lo contatta durante la raccolta dei dati e con il medico decidono il da farsi (16).

Il decision making nella gestione del paziente in Dialisi Peritoneale è stato esplorato nel setting di Nefrologia dell'ASMN - IRCCS di Reggio Emilia.

Le differenti modalità decisionali, tra novizi ed esperti, emerse dallo studio sono concordi con le principali caratteristiche descritte in letteratura.

Dal presente studio è in particolare emerso che il novizio, per la presa di decisione, a causa della mancata possibilità di confronto con situazioni simili, in un contesto altamente specialistico come la Nefrologia, può fare riferimento solo a nozioni generali che confronta con indicatori di monitoraggio rilevati sulla persona e, poiché non è in grado, sulla base dei dati, di fare ipotesi su possibili problemi/complicanze agisce quando il problema si è già manifestato. L'infermiere novizio, a causa della sua poca esperienza, alla comparsa del problema e dopo aver raccolto dati generali, chiama il medico.

Il professionista esperto, invece, in relazione alle ripetute esperienze con pazienti simili, è in grado, a partire dai dati disponibili o raccogliendone ulteriori, di fare ipotesi su possibili problemi/complicanze anticipando tutta una serie di interventi e, talvolta, persino interventi che richiederebbero una prescrizione medica.

\section{Conclusioni}

Lo studio contiene spunti di riflessione utili per l'identificazione di strategie che possano facilitare l'inserimento dell'infermiere novizio nel reparto di Nefrologia, ma anche per valorizzare meglio i saperi dell'esperto. A tal proposito, potrebbe essere utile, in studi futuri, implementare l'uso del piano standard per il paziente in dialisi peritoneale proposto, con la finalità di aiutarne la presa in carico da parte del novizio e di indagare ulteriormente, attraverso l'utilizzo dello strumento con un paziente reale, le differenti modalità di decision making tra infermiere esperto e novizio.

I limiti dello studio sono rappresentati dall'effettuazione dell'Audit durante il periodo di ferie estive, nel corso del quale erano assenti infermieri esperti e, dunque, c'erano infermieri "molto" neoassunti, ossia con pochi mesi di esperienza nel contesto di Nefrologia e anche nell'Azienda stessa. 
Il piccolo campione di infermieri, proveniente da un'unica struttura di Nefrologia e Dialisi, non consente la generalizzazione dei risultati a tutti i contesti di Nefrologia e Dialisi; tuttavia, quanto ottenuto dallo studio rappresenta una descrizione iniziale delle differenze che caratterizzano il decision making degli infermieri esperti e novizi in Nefrologia. Infine, non si ha la certezza che le azioni proposte in una situazione simulata siano le stesse che il professionista metterebbe in atto in una situazione reale.

\section{Disclosures}

Financial support: No financial support was received for this submission.

Conflict of interest: The authors have no conflict of interest.

\section{Bibliografia}

1. Conte G, Pacilio M, Garofalo C, Liberti ME, Provenzano M, Santangelo S. Epidemiologia della malattia renale cronica in Italia e strategie per la prevenzione. G Ital Nefrol. 2014;31(4): 2-10.

2. Kiberd B. The chronic kidney disease epidemic: stepping back and looking forward. J Am Soc Nephrol 2006;17(11):2967-73.

3. Meinero S, Alloatti S, Triolo G, et al. Non avvio o sospensione del trattamento dialitico cronico nell'adulto: considerazioni cliniche, relazionali, bioetiche e legislative. G Ital Nefrol. 2007;24(1):43-50.

4. Bonner A, Walker A. Nephrology nursing: blurring the boundaries: the reality of expert practice. J Clin Nurs. 2004;13(2):210218. Erratum in: J Clin Nurs. 2004;13(4):412.

5. Ugonia AP, Villa M. II ruolo degli infermieri nella dialisi peritoneale. G Ital Nefrol. 2013;30(1):1-8.

6. Kazancioglu R. Improving the quality of a peritoneal dialysis service: learning from experience. J Ren Care. 2013;39(Suppl.1):42-49.

7. Finkelstein FO, Ezekiel OO, Raducu R. Development of a peritoneal dialysis program. Blood Purif. 2011;31(1-3):121-124.

8. Bonner A. Recognition of expertise: an important concept in the acquisition of nephrology nursing expertise. Nurs Health Sci. 2003;5(2):123-131.

9. Bonner A, Greenwood J. The acquisition and exercise of nephrology nursing expertise: a grounded theory study. J Clin Nurs. 2006;15(4):480-489.
10. Bonner A. Uncovering the evidence of non-expert nephrology nursing practice. Int J Nurs Pract. 2006;12(2):51-56.

11. Bakalis NA, Watson R. Nurses decision-making in clinical practice. Nurs Stand. 2005;19(23):33-39.

12. Banning M. A review of clinical decision making: models and current research. J Clin Nurs. 2008;17(2):187-95

13. Aitken LM. Critical care nurses' use of decision-making strategies. J Clin Nurs. 2003;12(4):476-483.

14. Thompson C. A conceptual treadmill: the need for 'middle ground' in clinical decision making theory in nursing. J Adv Nurs. 1999;30(5):1222-1229.

15. Cioffi J. Nurses' experiences of making decisions to call emergency assistance to their patients. J Adv Nurs. 2000;32(1):108-114.

16. Bulfone G, Del Medico E, Blasotti F, Bresadola V. Le decisioni degli infermieri in chirurgia: quali differenze tra infermiere novizio ed esperto? Scenario. 2014;31(2):5-21.

17. Manias E, Aitken R, Dunning T. Decision-making models used by 'graduate nurses' managing patients' medications. J Adv Nurs. 2004;47(3):270-278.

18. Hedberg B, Sätterlund Larsson U. Observations, confirmations and strategies - useful tools in decision-making process for nurses in practice? J Clin Nurs. 2003;12(2):215-222.

19. Benner P. L'eccellenza nella pratica clinica dell'infermiere. L'apprendimento basato sull'esperienza. Milano, PA: McGrawHill 2003.

20. Benner P, Tanner C. Clinical judgment: how expert nurses use intuition. Am J Nurs. 1987;87(1):23-31.

21. Rew L. Acknowledging intuition in clinical decision making. J Holist Nurs. 2000;18(2):94-108,109-13.

22. King L, Clark JM. Intuition and the development of expertise in surgical ward and intensive care nurses. J Adv Nurs. 2002;37(4):322-329.

23. Watson S. An exploratory study into a methodology for the examination of decision making by nurses in the clinical area. $J$ Adv Nurs. 1994;20(2):351-360.

24. Effken JA. Informational basis for expert intuition. J Adv Nurs. 2001;34(2):246-255.

25. Hoffman K, Donoghue J, Duffield C. Decision-making in clinical nursing: investigating contributing factors. J Adv Nurs. 2004;45(1):53-62. .

26. Hancock HC, Easen PR. The decision-making processes of nurses when extubating patients following cardiac surgery: an ethnographic study. Int J Nurs Stud. 2006;43(6):693-705.

27. San Juan Miguelsanz MI, Muñoz Pilar S. Nephrology Nursing From basic training to specialist training. Enferm Nefrol vol.15 no.3 Madrid jul./sep. 2012 